\section{On the misguided use of reaction-time differences: A reply to Proctor and Rao (1982)}

\author{
ROGER RATCLIFF \\ Yale University, New Haven, Connecticut
}

and

\section{MICHAEL J. HACKER \\ Digital Equipment Corporation, Atlanta, Georgia}

Proctor and Rao (1982) present a discussion of our claim that models based on the difference in reaction time between positive and negative responses are essentially misguided. The experiments presented in Ratcliff and Hacker (1981) clearly show the effect of bias on the "same-different" reactiontime difference. Proctor and Rao reexamine the data presented and claim to find "same-different" differences that are invariant despite the bias manipulation. In this reply, we shall examine their reexamination of our data and discuss some further points concerning the theoretical interpretation of positivenegative response differences.

In Experiment 1 of Ratcliff and Hacker (1981), subjects performed a standard perceptual matching task. On each trial, subjects studied a string of letters and then were presented with a test string that was either identical to the study string or had $1,2,3$, or 4 letters replaced by new letters. The experimental variable of principle interest was the instruction to the subjects: they were to be cautious on either "same" responses or "different" responses (in different sessions). The main result was that the relative difference between reaction times for "same" and "different" responses changed from a 58 -msec advantage for "different" responses in the cautious "same" condition to a 110-msec advantage for "same" responses in the cautious "different" condition. Proctor and Rao point out that if one subtracts these two differences, one obtains $\mathbf{5 2} \mathrm{msec}$, which is the typical "same" advantage found in this task. They argue that this is an unbiased difference and conclude that, even though the relative speed of "same" and "different" responses is manipulable, one can still base models on this difference.

There is one assumption in this argument that should be made explicit. It is assumed that our bias manipulations were equally strong in the cautious "same" and cautious "different" conditions. We see

Preparation of this note was supported by NICHD Grant HD 16381 to Gail McKoon and Roger Ratcliff. R. Ratcliff's address is: Department of Psychology, Yale University, New Haven, Connecticut 06520. no reason for this assumption. One of the major points made in the whole tradeoff approach (be it signal detection theory or speed-accuracy tradeoff) is that one cannot assume an absolute criterion point. Subjects simply set criteria, and these may be biased more or less in one direction. Thus, from our point of view, this 52 -msec difference is simply a function of the relative bias attained by our particular subjects with these particular stimuli and nothing more. (Of course, there is still the problem of why the criterion is set where it is, but there are few quantitative answers to this question in other domains except when it can be shown that some function is optimized.)

Proctor and Rao also state that, in the matching literature, if anything, false "different" responses occur more often than false "same" responses. They argue that these results rule out any simple bias interpretation because a simple bias interpretation would require that along with fast "same" responses, more false "same" errors are produced. However, it should be noted that not all models of the matching process make the prediction that fast "same" responses require more false "same" responses than false "different" responses. For example, the model of Ratcliff (1981) has three independent criteria to be set by the subject, and by adjusting these criteria it is possible to produce more false "different" responses in conjunction with fast "same" responses.

For Proctor and Rao's procedure of subtracting the two reaction-time differences in Ratcliff and Hacker's Experiment 1 to be valid, error rates would have to show the pattern noted above, namely, either no difference or an advantage to false "different" responses. But, in fact, error rates are in the opposite direction: in the cautious "same" condition, the probability of a false "same" response is .076 and of a false "different," .109, whereas in the cautious "different" condition, the probability of a false "same" response is .139 and a false "different," .033. Thus, error rates invalidate Proctor and Rao's subtraction method and argue that the two bias conditions are not equivalent.

Proctor and Rao also discuss an experiment by Krueger and Shapiro (1981a; Experiment 3), in which single-letter stimuli were used and criteria were manipulated by varying the proportion of "same" and "different" trials that occurred in a block of trials. They report the same kind of "same" advantage as noted above and claim that this advantage supports their argument that even though the "same-different" bias may be adjusted, the fast "same" result still appears. However, a closer examination of Krueger and Shapiro's data indicates that this result should not be taken too seriously because of variability in the data. The variables that were manipulated were 
proportion of "same" and "different" responses and response-to-stimulus interval. When "same" judgments predominated, they were, on average, $33 \mathrm{msec}$ faster than "different" judgments, whereas when "different" judgments predominated, same judgments were $17 \mathrm{msec}$ faster than "different" judgments (a 16-msec difference). However, if the data are broken down by the four response-to-stimulus intervals, the differences (equivalent to the $16-\mathrm{msec}$ difference above) are $19,15,37$, and $-4 \mathrm{msec}$. The variability in this measure $(\mathrm{SE}=8.5 \mathrm{msec})$ indicates that the difference is not quite significant, so the conclusion should be viewed with caution.

In a second experiment, Ratcliff and Hacker (1981) introduced a new set of trials in which pairs of letters were transposed. On these trials, the test string was made up of the same letters as the study string, but two of the letters were transposed and subjects were required to respond "different". This manipulation changed the usual advantage for "same" responses over double-substitution "different" responses to a 77-msec disadvantage. Proctor and Rao argue that the discrimination of interest was no longer between "same" and substitution conditions, but between "same" and transposition conditions. Averaging the transposition conditions gives a "same" advantage of $46 \mathrm{msec}$. But this selection of one set of conditions for comparison without any further empirical or theoretical justification is not warranted. If one were to select all negative trials, there would be a $31-\mathrm{msec}$ disadvantage; on the other hand, if one were to select only the most difficult condition, there would be a 246-msec advantage.

So far, we have argued against Proctor and Rao's conclusions by noting problems in the interpretation of the data and by arguing that the criterion set between "same" and "different" responses is relative and that no absolute "unbiased" criterion setting exists. This is much the same as arguing that if subjects (operating with no biasing instructions) produce an asymmetry in hit and correct-rejection rates in an experiment measuring accuracy, this asymmetry should be treated as no more than a bias in criterion setting. The next step is to examine what aspects of data should be dealt with by a theory of matching. First, it is hard to press claims for some separate stage or process if models can fit the data adequately without such an assumption. For example, the models of Krueger (1978) and Ratcliff (1981) fit the data from a variety of matching experiments without assuming any additional stage for the usual "same" advantage (although, to be fair, Krueger and Shapiro, $1981 \mathrm{~b}$, have recently modified their position). Second, we suggest that there are more important aspects of the process to model than "same-different" reaction-time differences, namely, accuracy, reaction time distributions, and speed-accuracy functions. These kinds of data provide considerable constraints on the kinds of models that can be developed and provide relationships between different features of the matching process that cannot be examined by concentrating only on differences in mean reaction time.

In conclusion, we find Proctor and Rao's arguments unconvincing, and we suggest that models should not place too much weight on the difference between reaction time for positive and negative responses as an absolute measure of processing.

\section{REFERENCES}

Krueger, L. E. A theory of perceptual matching. Psychological Review. 1978, 85, 278-304.

Krueger, L. E., \& Shapiro, R. G. A reformulation of Proctor's unified theory for matching-task phenomena. Psychological Review, 1981, 88, 573-581. (a)

KrUeger, L. E., \& Shapiro, R. G. Intertrial effects of samedifferent judgments. Quarterly Journal of Experimental Psychology, 1981, 33A, 241-265. (b)

Proctor, R. W., \& Rao, K. V. On the "misguided" use of reaction-time differences: A discussion of Ratcliff and Hacker (1981). Perception \& Psychophysics, 1982, 31, 601-602.

RATCLIFF, R. A theory of order relations in perceptual matching. Psychological Review, 1981, 88, 552-572.

RAtClifF, R., \& HACKER, M. J. Speed and accuracy of same and different responses in perceptual matching. Perception \& Psychophysics, 1981, 30, 303-307.

(Manuscript received March 23, 1982; accepted for publication March 23, 1982.) 